\title{
GROWTH IN MATHEMATICAL UNDERSTANDING AND SPATIAL REASONING WITH PROGRAMMING ROBOTS
}

\author{
Krista Francis \\ University of Calgary \\ kfrancis@ucalgary.ca
}

\author{
Stefan Rothschuh \\ University of Calgary \\ kfrancis@ucalgary.ca
}

\author{
Brent Davis \\ University of Calgary \\ kfrancis@ucalgary.ca
}

This poster describes how programming robots might support both the development of spatial reasoning and growth in mathematical understanding using interpretive video analysis of two Grade 4 students' attempts to program their robot to follow a pentagon.

Theoretical Perspective. We argue that programming robots to move could lead to growth in mathematical understanding and contribute to developing spatial reasoning. We draw on Pirie and Kieren's (PK) (1994) model of growth in mathematical understanding which describes modes of engagement with mathematical concepts as seven distinct levels with increasing abstraction. We suspect that spatial reasoning is essential to all modes, but that it is especially relevant to the first three elements (primitive knowing, image making, and image having).

"[S]patial reasoning ... refers to the ability to recognize and (mentally) manipulate the spatial properties of objects and the spatial relations among objects" (Bruce et al., 2017, p. 147). Davis et al. (2015, p. 141) attempted to collect the many competencies and habits associated with spatial reasoning into a model that represents the emergent complexity of spatial reasoning skills as coevolved and complementary nature of the mental and physical actions.

Research Question. We questioned how programming robots might provide children with opportunities to gain mathematical understanding and develop spatial reasoning.

Data Collection Techniques and Analyses. Consistent with Knoblauch et al.'s (2013) notions of interpretive video analysis, we reviewed and selected one video based on instances of observable spatial engagement from 9 months of weekly videos collected of 32 Grade 4 students in 2 classrooms. In this video, a pair of students is attempting to program an EV3 LEGO Mindstorm robot to trace the third vertex of a pentagon having previous success following the first two straight-turn segments. We identified spatial elements in the two students' interactions according to Davis et al.'s (2015) framework while they engaged in determining how to steer their robot to travel around the $108^{\circ}$ vertex. We then analysed levels of mathematical understanding according to the PK model.

Summary of Findings. In this video one can observe the children working with many aspects of spatial reasoning and mathematical understanding. Drawing upon Davis et al.'s (2015) elements of spatial reasoning, the students were simultaneously INTERPRETING, [DE]CONSTRUCTING, MOVING, SITUATING, ALTERING and SENSATING. In the video, we observed the pair engage in how the distance the robot turns relates to the number of wheel rotations. The mathematical concepts included additive thinking, angles, properties of shape, measurement (distances, robot turns), multiplicative thinking (number of wheel rotations), pattern recognition, and direct proportion. Students' growth in understanding dynamically progressed between primitive knowing, image making, and image having. Our findings highlight how programming robots could support both the inner modes of PK's growth in mathematical understanding and contribute to developing spatial ability.

\section{References}

Bruce, C. D., Davis, B., Sinclair, N., McGarvey, L., Hallowell, D., Drefs, M., Francis, K., Hawes, Z., Moss, J., Mulligan, J., Okamato, Y., Whiteley, W., \& Woolcott, G. (2017). Understanding gaps in research networks: Using "spatial reasoning" as a window into the importance of networked educational research. Educational Studies in Mathematics, 95(2), 143-161. https://doi.org/10.1007/s10649-016-9743-2

In: Sacristán, A.I., Cortés-Zavala, J.C. \& Ruiz-Arias, P.M. (Eds.). (2020). Mathematics Education Across Cultures: Proceedings of the 42nd Meeting of the North American Chapter of the International Group for the Psychology of Mathematics Education, Mexico. Cinvestav / AMIUTEM / PME-NA. https:/doi.org/10.51272/pmena.42.2020 
Growth in mathematical understanding and spatial reasoning with programming robots

Davis, B., Okamoto, Y., \& Whiteley, W. (2015). Spatializing school mathematics. In Spatial reasoning in the early years: Principles, assertions, and speculations (pp. 139-150). Routledge.

Knoblauch, H., Tuma, R., \& Schnettler, B. (2013). Videography. Introduction to interpretive videoanalysis of social situations. Springer.

Pirie, S., \& Kieren, T. (1994). Growth in mathematical understanding: How can we characterise it and how can we represent it? Educational Studies in Mathematics, 26(2/3), 165-190. https://doi.org/10.1007/BF01273662. 